\title{
An Enhanced Accessory Pathway Localization Method for Efficient Treatment of Wolff-Parkinson-White Syndrome
}

\author{
Sándor M. Szilágyi ${ }^{1}$, László Szilágyi ${ }^{1,2}$, Levente K. Görög ${ }^{1}$, \\ Constantin T. Luca ${ }^{3}$, Dragoş Cozma ${ }^{3}$, Gabriel Ivanica ${ }^{3}$, and Zoltán Benyó ${ }^{2}$ \\ ${ }^{1}$ Sapientia - Hungarian Science University of Transylvania, \\ Faculty of Technical and Human Science, Târgu-Mureş, Romania \\ szs@ms.sapientia.ro \\ ${ }^{2}$ Budapest University of Technology and Economics, \\ Dept. of Control Engineering and Information Technology, Budapest, Hungary \\ ${ }^{3}$ Institute of Cardiovascular Medicine, University of Medicine and Pharmacy, \\ Timişoara, Romania
}

\begin{abstract}
This paper presents an analysis of the Arruda accessory pathway localization method for patients suffering from Wolff-ParkinsonWhite syndrome, with modifications to increase the overall performance. The Arruda method was tested on a total of 79 cases, and $91.1 \%$ localization performance was reached. After a deeper analysis of each decision point of the Arruda localization method, we considered that the lead aVF is not as relevant as other used leads (I, II, III, V1). The branch of the decision tree, which evaluates the left ventricle positions, was entirely replaced using different decision criteria based of the same biological parameters. The modified algorithm significantly improves the localization performance in the left ventricle. The overall performance reaches $94.9 \%$. A high localization performance of non-invasive methods is relevant because it can enlighten the necessary invasive interventions, and also reduces the discomfort caused to the patient.
\end{abstract}

Keywords: decision tree, sensitivity analysis, accessory pathway, WolffParkinson-White syndrome.

\section{Introduction}

The Wolff-Parkinson-White syndrome is characterized by an accessory pathway (by-pass tract) between the atria and ventricles that conducts parallel with the atrioventricular (AV) node - His bundle, but faster 1314. An accessory AV connection can conduct in both directions. The presence of these by-pass tracts may predispose to atria-ventricular reentrant tachycardia. Moreover, in the setting of atrial fibrillation, the WPW syndrome may cause a catastrophically rapid ventricular response with degeneration to ventricular fibrillation (VF). An adequate analysis of this phenomenon is important, since $0.1-0.2 \%$ of the population suffer from WPW syndrome [12. Unfortunately, the exact risk for developing VF during high ventricular rates is unknown. 
In the case of WPW syndrome, the electrocardiogram (ECG) tracing is a mixture of the electrical activities [1] caused by the accessory AV connection and normal AV conduction system. The fast impulse conduction produces an initial deflection in the QRS complex (delta wave) [11. The length of this delta wave is determined by the difference between the accessory AV connection and normal AV conduction times. The modified ventricular activation may cause secondary abnormalities in the ventricular repolarization such as: ST segment displacement (elevation or depression), $\mathrm{T}$ wave shape distortion and abnormal $\mathrm{U}$ wave. The modification of the conduction property of the accessory pathway (AcP), which may occur hour by hour or day by day, may cause alternating WPW pattern (complete, partial or missing pre-excitation, concertina effect).

Usually the WPW analysis is focused to develop and validate an accessory pathway localization method. A number of investigations have correlated ECG patterns and processing algorithms for detecting the place of the AcP 481, while other studies have been focused on the localization, realized through threedimensional heart reconstruction by the inverse solution of the ECG [10 5].

In this paper we present a sensitivity analysis of the Arruda's stepwise method [1], and a decomposition algorithm to improve the performance of AcP localization. Our main purpose is to detect more accurately the location of the ventricular insertion.

\section{Methods}

This research starts from the results and considerations published in 11]. There has been concluded, that the efficiency of the localization method - described in [1] by Arruda - depends significantly on the location of the AcP.

According to the above-mentioned studies, we developed a modified Arruda localization method, which was tested and evaluated through our measurements. The schematic representation of the research process can be seen in Fig. 1]

In the preliminary phase of the study we performed 12-lead ECG measurements for patients manifesting cardiac symptoms that served as a basis to select the subjects suffering from WPW syndrome (initial population - 85 persons). The ECG recordings were sampled at $500 \mathrm{~Hz}$ frequency with 12-bit resolution.

The standard diagnostic ECG usually comprises 12 leads: 3 bipolar standard leads (I - between left and right arm, II - between left leg and right arm, III between left leg and left arm), 3 unipolar limb leads (aVR, aVL, aVF), and six unipolar precordial leads (V1-V6) [12].

Our ECG signal processing concerned only accurate detection of the QRS complexes and delta $(\Delta)$ waves presented in Figure 2, constituting input parameters for the Arruda and modified Arruda algorithms.

As Fig. 3(a) presents, one cause for a wide QRS-complex that exceeds 0.12 seconds may be the Wolff-Parkinson-White syndrome (WPW syndrome). In this case the QRS-complex initially exhibits an early upstroke called delta wave. The two presented ECG signals differ significantly in shape. In both cases the interval 


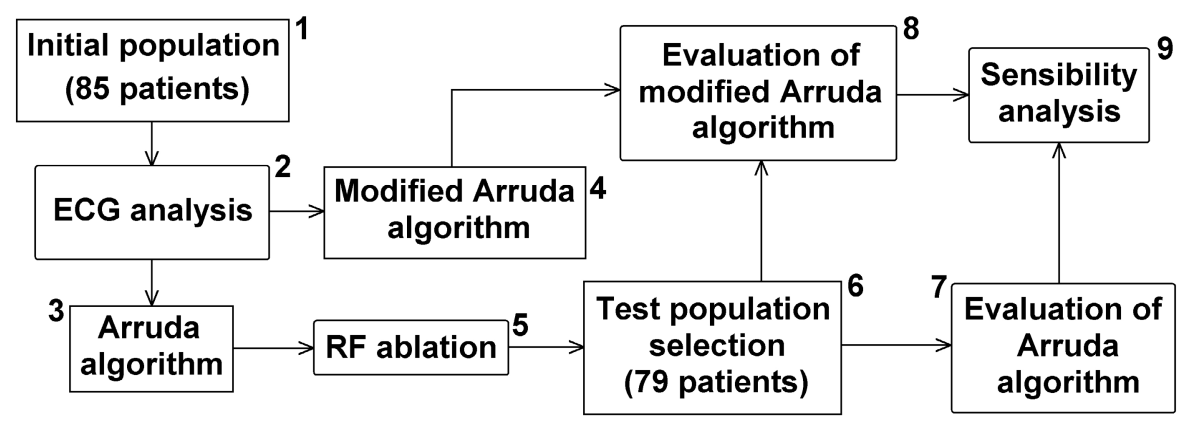

Fig. 1. Flow sheet of the research. The initial population (1) of the research consisted of 85 patients having at least one $\mathrm{AcP}$ (delta wave was detected in the preliminary phase of the study). For each patient a 5-10 minutes long 12-lead ECG recording was registered. The so-obtained data were then pre-processed and analyzed (2) in order to provide input for stages 3-4. Thereafter, we separately predicted the AcP locations by means of the Arruda (3) and modified Arruda (4) algorithms. After radio-frequency (RF) catheter ablation (5), 6 patients were excluded, due to multiple AcP's, while the remaining 79 ones were selected as test population (6) for further study phases. In the course of evaluation of Arruda (7) and modified Arruda (8) algorithms, the estimated positions of both localization methods (3-4) were evaluated one by one and validated with the outcome of RF ablation (5). Finally, within the frame of the sensitivity analysis (9), the results of stages 7-8 were statistically compared, followed by a vector-space dissection with regard to the spatial location of the ECG leads used by each algorithm.

from the $\mathrm{P}$-wave to the $\mathrm{R}$ spike is normal, but the early ventricular excitation forming the delta wave shortens the PQ-time.

The cause of the WPW syndrome is the transfer of activation from the atrium (region 1 in Fig. 3(a)) directly to the ventricular muscle via an abnormal route, called AcP, which bypasses the AV junctions. This partially activates the ventricular muscle (region 2 in Fig. 3(a)) before normal activation (region 3 in Figure 2) reaches it via the normal conduction system (after a delay in the AV junction). This process is called pre-excitation, and the resulting ECG depends on the specific location of the accessory pathway (see Fig. 3 (b)). With the help of localization methods the place of AcP can be determined.

The Arruda algorithm is a well known, clinically tested localization method, developed in order to identify the place of a singular accessory connection, reaching up to $90 \%$ recognition rate. It uses only five leads (I, II, III, aVF, V1) from the 12-lead ECG recordings. The first step of the algorithm is to analyze the amplitude relations of the $\mathrm{R}, \mathrm{S}$ and delta $(\Delta)$ waves in order to determine the AcP location (see Fig. 3(c)). The onset of the delta wave in each lead is measured from the onset of the earliest delta wave in any of the 12 leads. After $20 \mathrm{~ms}$ the displacement of the delta wave in each lead is classified as positive $(+)$, negative (-) or isoelectric (0) (see Fig. 3(c)), according to [7]. The 


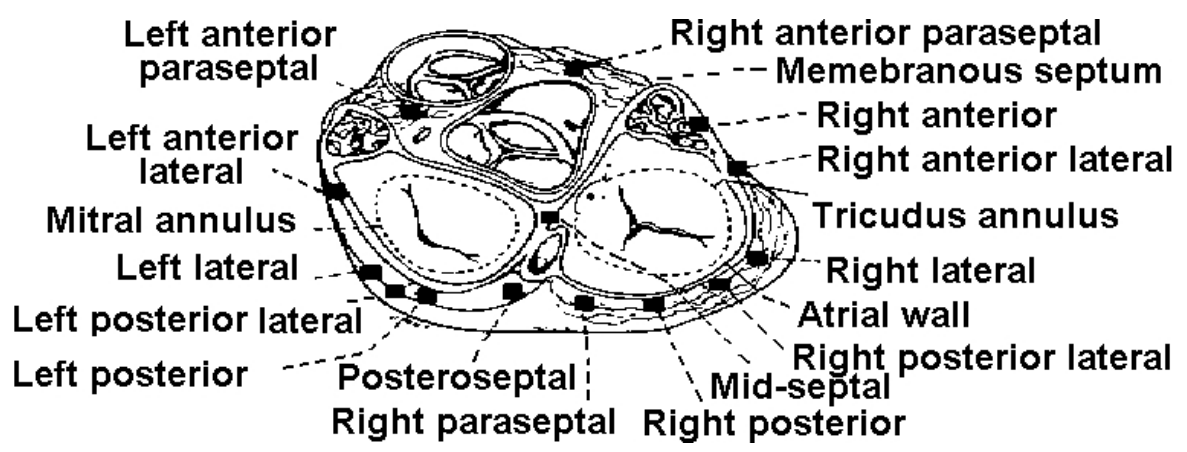

Fig. 2. Classification of the AcP pathways: (1) Septal accessory pathways: anteroseptal tricuspid annulus and right anterior paraseptal (AS/RAPS), mid-septal tricuspid annulus (MSTA), posteroseptal tricuspid annulus (PSTA), posteroseptal mitral annulus (PSMA), subepicardial posteroseptal (SEC); (2) Right free-wall accessory pathways: right anterior $(\mathrm{RA})$, right anterolateral $(\mathrm{RAL})$, right lateral $(\mathrm{RL})$, right posterolateral (RPL), right posterior (RP); (3) Left free-wall accessory pathways: left anterolateral (LAL), left lateral (LL), left posterolateral (LPL), left posterior (LP)

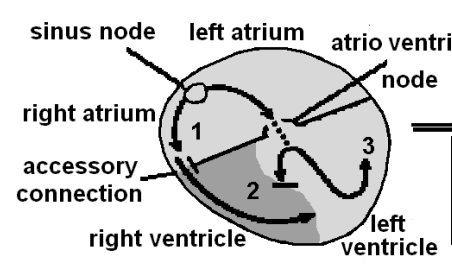

(a)

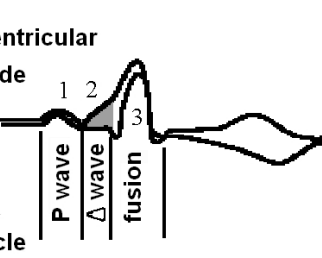

(b)

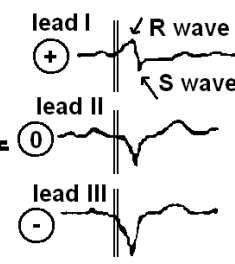

(c)

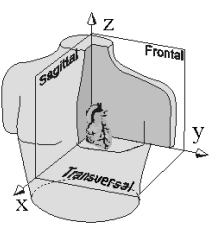

(d)

Fig. 3. (a)-(b) The emergence of WPW syndrome; (c) The delta wave polarity; (d) Conventional positions of $\mathrm{x}, \mathrm{y}$, and $\mathrm{z}$ axis

Arruda algorithm specifies a heart-region as the location of the AcP. According to Arruda, the possible AcP places have been divided into three major regions, which are further divided thereafter, as shown in Fig. 2. The proper Arruda algorithm might be interpreted as a decision tree consisting of simple two-way (YES/NO) ramifications, so called decision points (see Fig. 4). It has five leads (I, II, III, aVF, V1), R and S waves, respectively the displacement of the delta wave in each lead $(+,-, 0)$ as input, while the above mentioned heart-regions (locations) as output. Although it has been statistically concluded that the performance of the Arruda algorithm is about $90 \%$, some modifications in this localization method could be beneficial. In [11] we pointed out that in most of the cases the estimation errors of the Arruda algorithm are correlated with aVF sign test. In the same place we suggested modifying the lead aVF where it is possible.

The modified Arruda algorithm (Fig. 4) developed in order to identify the place of a singular accessory connection uses the same leads, same heart regions 


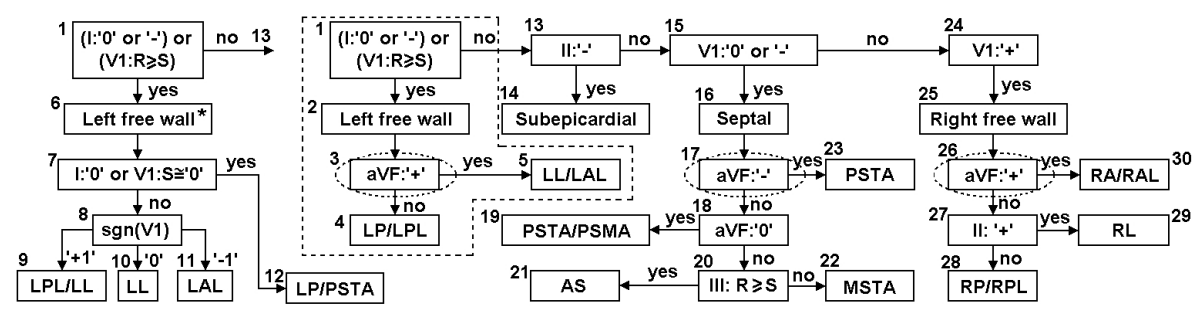

Fig. 4. Presentation of the Arruda and modified Arruda localization method. The block encircled with dashed line is replaced with the left ventricular branch shown on the left side of the diagram.

for localization of the AcP and same displacement of the delta wave in each lead $(+,-, 0)$ as Arruda. The new algorithm remains a decision tree consisting of simple two-way (yes/no) ramifications, with one exception at place 8. Although [11] revealed that the most sensible aVF sign tests at locations 3,17 and 26, we modified only the first one at left free wall. The other two sensible places are marked as places 17 and 26. The left free wall region is completely altered for better performance but all other tests are the same as in Arruda's work.

RF catheter ablation of cardiac tissue is highly effective in the invasive treatment of WPW syndrome [3. In the course of RF ablation, a priori knowledge about the target ablation site(s) could be beneficial due to shorter intervention time and therefore lower risk. The Arruda (as well as the modified Arruda) algorithm predicts the location of a singular $\mathrm{AcP}$, serving as a starting point to the surgeon. However, the exact number and place of the AcP-s will be determined only during the surgical operation. Therefore, RF ablation plays a validation role for both localization methods. By means of RF ablation, we excluded from the research 6 patients with multiple AcP-s, thus obtaining a test population consisting of 79 subjects.

\section{Results and Discussion}

To evaluate the performance of a localization method we had to analyze the relationship between the predicted location (based upon the ECG algorithm) and the real location (based upon ablation site) of the AcP.

Table 1 and Table 2 present this relation for both algorithms, indicating the sensitivity and specificity for all AcP locations. Ablation sites are represented in vertical and the predicted locations in horizontal direction.

All AcP location predictions were analyzed in order to determine the strong and weak decision points of both localization methods. During performance determination the contribution of each decision point was taken into consideration in when it was possible. In some cases a failed decision made all further evaluation meaningless. Such cases are indicated having no result. The last column shows the decision performance for all possible algorithmic branches. Table 3 presents the global accuracy of each decision point. A penetrative study of the 
Table 1. Detection accuracy of the accessory pathway with Arruda localization method

\begin{tabular}{|c|c|c|c|c|c|c|c|c|c|c|c|}
\hline$\overline{\text { Ablation site }}$ & Number & 4. & 5. & 14. 19. & $\overline{21.2}$ & 2.23 . & 28. & 29 . & 30 & Sens. & Spec. \\
\hline 4. LP/LPL & 2 & 1 & & 1 & & & & & & 50.0 & 96.2 \\
\hline 5. LL/LAL & 22 & 2 & 20 & & & & & & & 90.9 & 100.0 \\
\hline 14. SEC & 10 & & & 10 & & & & & & 100.0 & 100.0 \\
\hline 19. PSTA/PSMA & 1 & & & 1 & & & & & & 100.0 & 98.7 \\
\hline 21. AS/RASP & 2 & & & & 2 & & & & & 100.0 & 100.0 \\
\hline 22. MSTA & 3 & & & & 3 & & & & & 100.0 & 98.7 \\
\hline 23. PSTA & 14 & 1 & & & 1 & 12 & & & & 85.7 & 100.0 \\
\hline 28. RP/RPL & 6 & & & & & & 5 & & 1 & 83.3 & 100.0 \\
\hline 29. RL & 8 & & & & & & & 7 & 1 & 87.5 & 100.0 \\
\hline 30. RA/RAL & 11 & & & & & & & & 11 & 100.0 & 97.1 \\
\hline$\overline{\text { All }}$ & 79 & & & & & & & & & 91.1 & 99.0 \\
\hline
\end{tabular}

Table 2. Detection accuracy of the accessory pathway with modified Arruda localization method

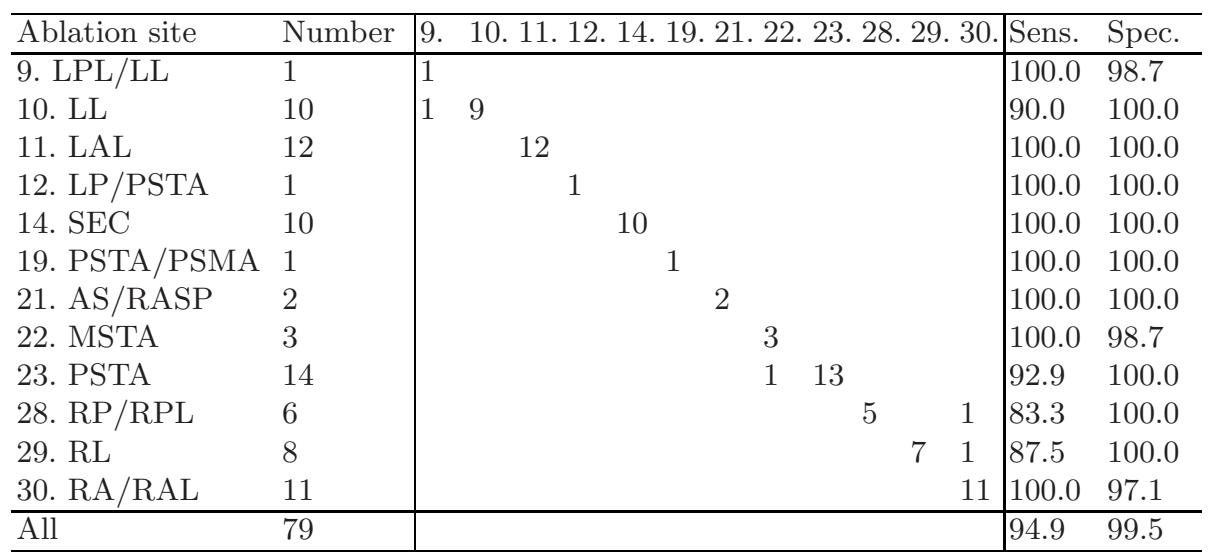

Table 3. Global accuracy of each decision point for both Arruda and modified Arruda localization method

\begin{tabular}{ccccrr}
\hline Decision no. & Total decisions & \multicolumn{2}{c}{ Failed decisions } & \multicolumn{2}{c}{ Performance } \\
\hline 1 & & Arruda & Modified & Arruda & Modified \\
\hline 3 & 79 & 1 & 0 & $98.73 \%$ & $100.00 \%$ \\
7 & 24 & 2 & N/A & $91.67 \%$ & N/A \\
8 & 24 & N/A & 0 & N/A & $100.00 \%$ \\
13 & 23 & N/A & 1 & N/A & $95.65 \%$ \\
15 & 54 & 0 & 0 & $100.00 \%$ & $100.00 \%$ \\
17 & 44 & 0 & 0 & $100.00 \%$ & $100.00 \%$ \\
18 & 20 & 1 & 1 & $95.00 \%$ & $95.00 \%$ \\
20 & 6 & 0 & 0 & $100.00 \%$ & $100.00 \%$ \\
26 & 5 & 0 & 0 & $100.00 \%$ & $100.00 \%$ \\
27 & 25 & 2 & 2 & $92.00 \%$ & $92.00 \%$ \\
\hline
\end{tabular}


localization method demands much more, than a simple evaluation of its overall performance, which proves to depend mostly on the least efficient decision points. A deeper investigation of Table 3 reflects that applying Arruda localization method the estimation errors often (about $80 \%$ of the cases) appear due to wrong decision at places 3, 17, 26 (see Fig. 4). All of these decision points contain an aVF sign test. Furthermore, it is observable that in the diagram none of the aVF sign tests perform reliably.

The main goal of the modified localization method was to eliminate the most sensible decision points and thus to increase the overall performance. However, not all sensible decisions of the Arruda algorithm can be wisely replaced, based on the 12-lead ECG, due to the followings:

- The depolarization wave's sphere-symmetric propagation is highly deformed by the fact, that the right ventricle wall $(4-5 \mathrm{~mm})$ is much thinner than the left $(10-12 \mathrm{~mm})$ or the septal $(7-8 \mathrm{~mm})$ one. Taking into consideration the fact, that the depolarization wave propagates at $300-800 \mathrm{~mm} / \mathrm{sec}$, and the sign of the delta wave is investigated over 20-millisecond intervals, it is obvious that vectorial calculations cannot accurately describe the behavior of the right ventricle.

- The three leads used by Arruda, namely I, V1 and aVF, are almost orthogonal. Although V2 should perform better than V1, no significant difference proved achievable. Unfortunately we have to admit, that despite Arruda's decision points 10 and 19, which detect the septal and right regions, are sensible, they cannot be wisely replaced within the scope of the 12 lead ECG. This is illustrated also in Fig. 4, where the aVF tests are encircled to indicate the necessity of further development.

The thicker wall of the left ventricle allows us to apply vectorial analysis, therefore in this region significant improvements are possible.

Arruda's leads I and aVF (decision points 1 and 3) do not assure visibility in the direction of $\mathrm{x}$ axis (see Fig. 3(d)). The modified method (decision points 1, 7 and 8) uses the $\mathrm{z}$ direction instead, which according to Table 3 at least partially improves the performance. Decision point 8 needs further refinement, because its $95 \%$ accuracy is relatively low. The accessory pathway localization requires a robust decision tree solution with precise yes/no criteria, due to its clinical application which accepts no fuzzy decisions.

\section{Conclusions}

Finally we can conclude, that Arruda's leads used for the detection of septal and right regions seem to give optimal solution in the scope of 12-lead ECG. The proposed modification has reduced the mistaken detections of the accessory pathway in the left ventricle by $80 \%$. The about $95 \%$ recognition rate we considered quite good, but a straightforward comparison with other localization methods [2] performed on the same database would be beneficial in order to create a better noninvasive localization method [1]. A high localization performance of non-invasive 
methods is relevant because they can enlighten the necessary invasive interventions, and also reduce the discomfort caused to the patient.

\section{References}

1. Arruda, M.S., McClelland, J.H., Wang, X., Beckman, K.J., Widman, L.E., Gonzalez, M.D., Nakagawa, H., Lazzara, R., Jackman, W.H.: Development and validation of an ECG algorithm for identifying accessory pathway ablation site in Wolff-Parkinson-White syndrome. J. Cardiovasc. Electrophys. 9, 2-12 (1998)

2. Boersma, L., Moran, E.G., Mont, L., Brugada, J.: Accessory pathway localization by QRS polarity in children with Wolff-Parkinson-White syndrome. J. Cardiovasc. Electrophys. 13, 1222-1226 (2002)

3. Cao, H., Vorperian, V.R., Tsai, J.Z., Tungjitkusolmun, S., Woo, E.J., Webster, J.G.: Temperature measurement within myocardium during in vitro RF catheter ablation. IEEE Trans. Biomed. Eng. 47, 1518-1524 (2000)

4. Fitzpatrick, A.P., Gonzales, R.G., Lesh, M.D., Modin, G.W., Lee, R.J., Scheinmen, M.M.: New algorithm for the localization of accessory atrioventricular connections using a baseline electrocardiogram. J. Am. Coll. Cardiol. 23, 107-116 (1994)

5. Guanglin, L., Bin, H.: Localization of the site of origin of cardiac activation by means of a Heart-Model-Based electrocardiographic imaging approach. IEEE Trans. Biomed. Eng. 48, 660-669 (2001)

6. Guize, L., Soria, R., Chaouat, J.C., Chrétien, J.M., Houe, D., Le Heuzey, J.Y.: Prevalence and course of Wolff-Parkinson-White syndrome in population of 138,048 subjects. Ann. Med. Int. Paris 136, 474-489 (1985)

7. Levkov, C., Mihov, G., Ivanov, R., Daskalov, I., Christov, I., Dotsinsky, I.: Removal of power-line interference from the ECG: a review of the subtraction procedure. BioMedical Engineering OnLine 4(50), 1-18 (2005)

8. Reddy, G.V., Schamroth, L.: The localization of bypass tracts in the WolffParkinson-White syndrome from the surface electrocardiogram. Am. Heart J. 113, 984 (1987)

9. Rosenbaum, F.F., Hecht, H.H., Wilson, F.N., Johnston, F.D.: The potential variation of the thorax and the esophagus in anomalous antrioventricular excitation (Wolff-Parkinson-White syndrome). Am. Heart J. 29, 281-326 (1945)

10. Shahidi, A.V., Savard, P., Nadeau, R.: Forward and inverse problems of electrocardiography: modeling and recovery of epicardial potentials in humans. IEEE Trans. Biomed. Eng. 41, 249-256 (1994)

11. Szilágyi, S.M., Benyó, Z., Frigy, A.: Sensibility Analysis of the Arruda Localization Method. Sci. Bull. Univ. Timişoara, Ser. Aut. Electr. Eng. 49(63), 129-132 (2004)

12. Wellens, H.J.J., Fare, J., Bar, F.W.: The Wolff-Parkinson-White syndrome. In: Mandel, W.J. (ed.) Cardiac arrhythmias. Their mechanisms, diagnosis and management, pp. 274-296. JP Lippincott, Philadelphia (1987)

13. Wolff, L., Parkinson, J., White, P.: Bundle branch block with short PR interval in healthy young people prone to paroxysmal tachycardia. Am. Heart J. 5, 685-704 (1930)

14. Yee, R., Klein, G.J., Guiraudon, G.M.: The Wolff-Parkinson-White Syndrome. In: Zipes, D.P., Jalife, J. (eds.) Cardiac electrophysiology: from cell to bedside, pp. 1199-1214. WB Saunders Co., Philadelphia (1995) 